\title{
Long-term outcomes of laparoscopic sleeve gastrectomy - a single-center prospective observational study
}

\author{
Radu Neagoe ${ }^{1}$, Mircea Muresan ${ }^{1}$, Daniel Timofte ${ }^{2}$, Ruxandra Darie ${ }^{1}$, Ion Razvan ${ }^{1}$, Septimiu Voidazan ${ }^{3}$, \\ Simona Muresan ${ }^{4}$, Daniela Sala ${ }^{1}$ \\ ${ }^{1}$ Surgery Clinic No. 2, University of Medicine, Pharmacy, Science and Technology, Targu Mures, Romania \\ ${ }^{2}$ Surgery Clinic No. 3, University of Medicine and Pharmacy Gr.T.Popa, Iasi, Romania \\ ${ }^{3}$ Epidemiology Department, University of Medicine, Pharmacy, Science and Technology, Targu Mures, Romania \\ ${ }^{4}$ Physiology Department, University of Medicine, Pharmacy, Science and Technology, Targu Mures, Romania
}

Videosurgery Miniinv 2019; 14 (2): 242-248 DOI: https://doi.org/10.5114/wiitm.2019.84194

\begin{abstract}
Introduction: Laparoscopic sleeve gastrectomy (LSG) is an established bariatric procedure.

Aim: We present our long-term results regarding weight loss and comorbidities during 9 years.

Material and methods: We calculated the percent excess weight loss (\%EWL) and changes in body mass index $(\triangle B M I)$. We evaluated arterial hypertension (AHT), type 2 diabetes (T2DM) and obstructive sleep apnea syndrome (OSAS).

Results: One hundred seventy-nine patients were included (136 female/43 male), mean age of $40.47 \pm 11.08$ years, median preoperative body mass index (BMI) of $42.93 \mathrm{~kg} / \mathrm{m}^{2}$. Median follow-up period was 72 months (36-84 months). The \%EWL during follow-up was 41.8 ( $n=179$ patients, at 3-month follow-up), $64.1(n=163), 75.33(n=$ 134), $77.1(n=103), 76,03(n=99), 73.78(n=64), 71.58(n=37), 63.83(n=22)$ and $64.1(n=14)$ at $6,12,18,24$, $36,48,60$ and 72 months, respectively. We noted a negative correlation between \%EWL and both the age and initial weight and BMI of the patient; a negative correlation between gender (male patients) and \%EWL was also found. After LSG, 68.2\% of patients with AHT presented resolution (no medication) or significant improvement (doses reduced) of the disease. As regards T2DM, 65.8\% described resolution or significant improvement after surgery. Furthermore, 31 (70.4\%) patients with preoperative OSAS reported resolution/improvement within a year from surgery. Conclusions: Laparoscopic sleeve gastrectomy is a safe and effective procedure, with good results in the short and medium term. Long-term follow-up reveals a tendency to weight regain after approximately 2 years from primary surgery, with the need for revisional surgery in some cases.
\end{abstract}

Key words: laparoscopic sleeve gastrectomy, long-term follow-up, weight regain.

\section{Introduction}

During recent years, as long as the obesity epidemic grew, bariatric surgery evolved and became a standalone surgical specialty, the number of procedures increasing annually. Laparoscopic sleeve gastrectomy (LSG) is now recognized by the majori- ty of authors as the most accepted and widespread bariatric technique [1]. Several randomized trials [2, 3] and large series studies [4] confirmed that LSG is a safe and effective operation, in terms of both weight reduction and control of the main obesity comorbidities. Different consensus conferences have tried, and mostly managed, to answer a series 
of questions that persist regarding this particular intervention [5], so at the time, we can talk about some standardization of the surgical technique. However, many aspects remain debatable, including those concerning long-term evolution of patients, maintaining weight loss and the beneficial effect on obesity-related comorbidities. In Romania, as in other Eastern Europe countries, LSG has gained substantial ground ahead other bariatric operations, i.e. vertical banded gastroplasty (VBG) and laparoscopic adjustable gastric banding (LAGB), over the past two decades. Even though throughout the years, centers of excellence dedicated to bariatric surgery have emerged, they are relatively few when compared to the high prevalence of obesity in the geographic region. Moreover, there are still only a few studies on large groups of patients including medium- and long-term follow-up.

\section{Aim}

The aim of this retrospective study is to bridge a gap in the autochthonous literature regarding long-term results after LSG, by assessing a single surgeon's experience in an Eastern Europe university centre, with more than 200 cases operated on and up to 7 years of follow-up.

\section{Material and methods}

\section{Study design}

A single surgeon database was revised for the records of patients who underwent LSG as a primary bariatric procedure between October 2010 and March 2018. We gathered information regarding patient characteristics, certain laboratory parameters, details on the surgery, and perioperative and postoperative complications; the postoperative evolution of the weight curve and the effect of surgery on the main comorbidities were followed as well. The inclusion criteria were set according to the recommendations of the National Institutes of Health 1991 Bariatric Surgery Guidelines and the Romanian Surgical Society; we excluded patients who had other types of bariatric surgery than LSG, patients with LSG but operated on by other surgeons in our department, as well as those with less than 3 months of follow-up; patients who we were not able to contact and thus did not respond to our questionnaire were also excluded. All patients were informed about the procedure and possible complications, and informed consent was obtained. This study meets the STROBE criteria (STrengthening the Reporting of OBservational studies in Epidemiology) and has been approved by the Hospital's Ethics Committee (number 4065/2014).

The patients' assessment was made by a multidisciplinary team and included upper gastrointestinal endoscopy, abdominal ultrasonography, and sleep apnea testing - whenever obstructive sleep apnea syndrome (OSAS) was encountered. Our thromboprophylaxis protocol consisted in preoperative administration of low molecular weight heparin (LMWH) along with other already acknowledged measures, such as graduated compression stockings or a sequential compression device.

\section{Surgical technique}

Every procedure was performed by laparoscopy and four to six trocars were used. A $34 \mathrm{Fr}$ bougie was used to calibrate the sleeve and 5-7 firings were usually required. In approximately half of the patients, we performed over-sewing as a reinforcing method; the rest of the cases had no reinforcement, the hemostasis being achieved by placing clips on visible bleeding sites of the staple line. We had patients with concomitant procedures, mainly posterior cruroplasties for associated hiatal hernias. Methylene blue solution was used to assess the staple line and a silicon drain was left on site beside the resected stomach for the following 24-36 h.

\section{Follow-up}

We prospectively followed up our patients at a 3-month period, for the first year after LSG and then twice a year; further information was collected through e-mail, written correspondence (including social media) or by phone. Every patient filled out a questionnaire; the entire weight loss process was recorded and any discontinuance or decrease of drug dosage was considered an achieved remission or amelioration of the main comorbidities.

\section{Evaluation of outcomes}

To measure the effectiveness of LSG we calculated the percentage excess weight loss (\%EWL) and change in body mass index $(\triangle B M I)$. The obtain the excess weight we subtracted the ideal body mass (BMI $=25 \mathrm{~kg} / \mathrm{m}^{2}$ ) from the initial body weight (measured at the time of hospital admission). The percentage of 
excess weight loss (\%EWL) was calculated according to the following formula: $[100 \times$ (preoperative body weight - present body weight)/excess weight]. Satisfactory weight loss after surgery was defined by $\%$ EWL greater than $50 \%$. We evaluated arterial hypertension (AHT) and OSAS based on investigations and postoperative modification in pharmacological therapy. As regards type 2 diabetes mellitus (T2DM) assessment, we did not have information concerning postoperative glycosylated hemoglobin $\left(\mathrm{HbA}_{1 \mathrm{c}}\right)$ in an important number of patients; thus we interpreted the results in the same subjective manner - whether reduction in doses (improvement) or cessation of treatment (resolution) was observed; this aspect is noted below as a limitation of the study.

\section{Statistical analysis}

All statistical calculations were performed using Graph Pad Software, San Diego, California, USA. We tested the normal distribution for continuous variables using the Kolmogorov-Smirnov test. We char-

Table I. Preoperative and operative characteristics of study patients

\begin{tabular}{|lc|}
\hline Parameter & Value \\
\hline Age, mean \pm SD [years]: & $40.47 \pm 11.084$ \\
\hline Age $>60$ years, $n(\%)$ & $9(0.05)$ \\
\hline Sex (F/M), $n$ (\%) & $136 / 43(75.9 / 24)$ \\
\hline Weight, median (range) [kg] & $120(90-216)$ \\
\hline BMI, median (range) $\left[\mathrm{kg} / \mathrm{m}^{2}\right]:$ & $42.93(33-73)$ \\
\hline BMI $>50 \mathrm{~kg} / \mathrm{m}, n(\%)$ & $39(21.8)$ \\
\hline EBW, median (range) $[\mathrm{kg}]$ & $50.2(23.5-142)$ \\
\hline Waist circumference, mean \pm SD [cm] & $121.21 \pm 17.54$ \\
\hline Comorbidities, $n$ (\%): & $85(47.4)$ \\
\hline Hypertension & $41(22.9)$ \\
\hline Diabetes & $44(24.5)$ \\
\hline OSAS & $51(28.4)$ \\
\hline Dyslipidemia & $28 / 19(15.6 / 10.6)$ \\
\hline Minor GERD/associated HH & $41(22.9)$ \\
\hline Synchronous procedures, $n$ (\%) & $0(0)$ \\
\hline Conversion to open, $n$ (\%) & $131(67-241)$ \\
\hline Operative time, median (range) [min]: & $92(57-176)$ \\
\hline First 50 procedures & $3(2-9)$ \\
\hline After 50 procedures & \\
\hline Hospital stay, median (range) [days] & \\
\hline
\end{tabular}

$B M I$ - body mass index, EBW - excess body weight, OSAS - obstructive sleep apnea syndrome, GERD - gastroesophageal reflux disease. acterized them as mean and standard deviation (SD) for variables with normal distribution, or as median and range for variables with non-normal distribution. We chose appropriate statistical tests according to data distribution. The difference in mean age by gender was determined by Student's $t$-test. Differences between values of compared preoperative and postoperative variables ( 3 months and 6 years, respectively) were determined by ANOVA test (associated with the Bonferroni multiple comparison test). The correlation between quantitative variables was assessed using Pearson correlation, when appropriate. Statistical significance was set at $p<0.05$.

\section{Results}

Two hundred twenty-four consecutive patients underwent LSG in the study period; 22 patients were excluded because they had a follow-up less than 3 months; furthermore, we were unable to contact 23 for different reasons. Finally, the study population comprised 179 patients, 136 women and 43 men, with an average age of 40 years and a median BMI of $43 \mathrm{~kg} / \mathrm{m}^{2}$; we had 9 patients over 60 years and $21.8 \%$ with a BMI > $50 \mathrm{~kg} / \mathrm{m}^{2}$. The main general characteristics of the patients are presented in Table I.

We did not record any case of conversion to open surgery; the operating time diminished significantly after the first 50 procedures $(p=0.001)$, and the number of procedures performed annually has increased constantly over the last 3 years. We used a median of 6 (range: $5-7$ ) cartridges and a buttress was only used in the first cases that we operated on. Otherwise, reinforcement was performed in 79 $(44.1 \%)$ of the cases by oversewing, which was not done in all cases on the entire length of the staple line. In 92 (52.2\%) cases, no reinforcement was performed, but only targeted hemostasis at the level of the staple line using metallic clips. Concomitant procedures were performed in 41 (22.9\%) cases, most of them being posterior crural repairs for associated hiatal hernia $(n=19)$; other synchronous procedures were cholecystectomies $(n=7)$, adhesiolysis $(n=6)$, and parietal hernia $(n=4)$. We also had 8 cases of adjustable gastric band removal, including 2 cases of intragastric band migration, where a laparoscopic transgastric removal of the eroded adjustable band was done; for 5 of these patients conversion to LSG was performed at the time. The overall early morbidity (< 30 days) was $11.1 \%$ with a $4.4 \%$ rate of 
major complications; we had 6 cases of postoperative hemorrhage with 5 laparoscopic reinterventions, the majority of which had the source at the level of the staple line $(n=4)$. We also encountered a proximal leak, 6 days after the surgery; initially, a laparoscopic drainage and a feeding-jejunostomy were performed, and then the fistula was closed endoscopically 4 months after the primary surgery. Two (0.01\%) patients developed an intra-abdominal abscess; for these a laparoscopic drainage was performed. Two cases presented late stenosis; one case responded favorably to endoscopic dilation and the other required reintervention 5 months after LSG when a anastomosis gastric bypass (OAGB) was performed. Mortality in our group was $0.005 \%$ $(n=1)$, in a patient who developed a subphrenic and left pleural abscess 6 months after the primary intervention, probably due to a late fistula.

After LSG, 33 (18.4\%) patients presented gastroesophageal reflux disease (GERD) de novo or an aggravation of the preoperative minor reflux symptoms; in 3 cases a posterior cruroplasty was done within 1 year after LSG and 3 other patients had their LSG converted to LGB. Late reinterventions (> 30 days) were also performed for biliary lithiasis $(n=7)$, antral stenosis $(n=1)$, and incisional port-hernia $(n=1)$. We also had 16 (8.9\%) patients who had sleeve revision for weight regain and for whom several types of laparoscopic bariatric reintervention were performed: 7 LGB (3 Roux en Y gastric bypasses and 4 OAGB), 6 re-gastric sleeve, 3 banded-sleeve.

The median follow-up was 18 months (3-72 months). We lost 21 patients to follow-up, resulting in a follow-up rate of $88 \%$; these patients were excluded from the weight-loss analyses. The weight loss process during follow-up, expressed as \%EWL and $\triangle B M I$, is shown in Table II. We noted significant increases between mean values in the first 2 postoperative years ( $p=0.0001)$; afterwards the differences did not reach statistical significance, while the \%EWL and $\triangle B M I$ decreased towards the final follow-up period.

We established several correlations between preoperative demographic and anthropomorphic characteristics and \%EWL; thus, we noted a negative correlation between \%EWL and both the age (\%EWL being more reduced in the case of elderly patients) and initial weight and $\mathrm{BMI}$ of the patients (better weight loss in the low BMI group, under $45 \mathrm{~kg} / \mathrm{m}^{2}$ ). In addition we found a negative correlation between gender and \%EWL with a significantly better weight loss in the female group (Table III).

Before surgery, 85 (47.4\%) patients were found with $\mathrm{AHT}$ and 41 (22.9\%) with T2DM; we had only $24.5 \%$ of our patients with preoperative OSAS. After LSG, $68.2 \%$ of patients with AHT presented resolution (no medication) or significant improvement (doses reduced) of the disease. As regards T2DM, 65.8\% described resolution or significant improvement after surgery. Furthermore, 31 patients (70.4\%) with preoperative OSAS reported resolution/ improvement of this disease, confirmed by polysomnography performed within a year from surgery.

\section{Discussion}

Herein we present in a retrospective view the experience of a single surgeon, who introduced and developed bariatric surgery in a tertiary university center in Romania, the initial experience having been previously reported [6]. We found that LSG is a safe procedure with low postoperative morbidity and mortality rates. We also established that LSG is effective bariatric surgery, especially in certain groups of patients, even if a tendency toward weight

Table II. Weight loss effect

\begin{tabular}{|c|c|c|c|c|c|c|c|c|c|}
\hline \multirow[t]{2}{*}{ Parameter } & 3 months & 6 months & 1 year & 1.5 years & 2 years & 3 years & 4 years & 5 years & 6 years \\
\hline & 179 & 163 & 141 & 103 & 99 & 64 & 37 & 22 & 14 \\
\hline \multicolumn{10}{|l|}{ \%EBW: } \\
\hline Mean & 41.84 & 64.18 & 75.33 & 77.13 & 76.03 & 73.79 & 71.58 & 63.83 & 64.10 \\
\hline Standard deviation & 16.51 & 21.18 & 16.97 & 16.91 & 18.03 & 20.35 & 18.60 & 19.00 & 22.56 \\
\hline \multicolumn{10}{|l|}{$\Delta \mathrm{BMI}:$} \\
\hline Mean & 7.306 & 11.30 & 15.34 & 16.65 & 16.39 & 16.08 & 15.03 & 12.73 & 14.28 \\
\hline Standard deviation & 2.502 & 3.089 & 4.134 & 5.558 & 5.869 & 6.499 & 4.598 & 4.697 & 6.621 \\
\hline
\end{tabular}

Data are expressed as mean \pm SD. ANOVA and Bonferroni multiple comparison test. 


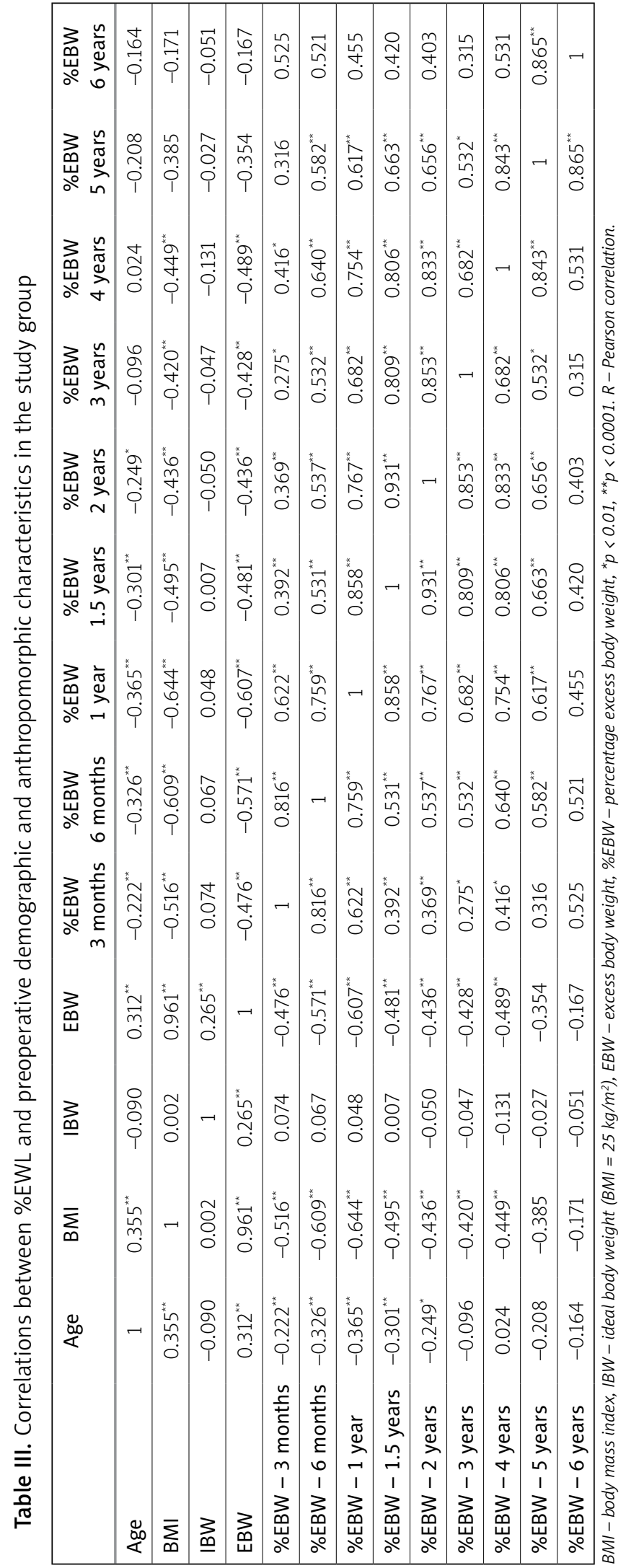

regain is described on long-term follow-up, with the need for redo surgery in some cases.

Surgical technique is mostly standardized, although some aspects remain controversial. In the first cases we actually performed a longitudinal "vertical" resection, starting it at approximately $6 \mathrm{~cm}$ from the pylorus; this technique is still preferred by some authors [7, 8] based on the practical consideration of preserving the contractile function of the antrum. Subsequently, we modified the technique and prepared the greater curvature up to $2-3 \mathrm{~cm}$ from the pylorus, partially including the antrum in the resection, so that the final form of the sleeve is an "L" shape. This technical detail has been previously described in the literature $[9,10]$, its purpose being primarily to reduce the sleeve size. Although recent meta-analyses and reviews suggest the reinforcement role in reducing occurrence of leaks and other associated complications $[11,12]$, this is not yet considered standard for LSG. For a significant number of patients we used oversewing as a reinforcing method, having the impression of good hemostasis and even a further tightening of the resected stomach; in these cases the stapling had to respect a minimal distance from the bougie, in order to allow an inverting suture. For the rest of the cases, we did not use any reinforcement, stapling close to the bougie and using metal clips for targeted hemostasis, where necessary. We did not comparatively analyze these groups of patients, i.e. oversewing vs no oversewing, vertical sleeve vs. "L" shape resection, a limitation of our study which is admitted below.

The GERD was initially considered only a relative contraindication for LSG $[5,13]$. However, recently published long-term follow-up studies confirmed postoperative aggravation of GERD and Barrett's esophagus after LSG [14-17]. At the beginning of our study we assessed GERD only through endoscopy, which obviously is not the gold standard GERD diagnosis, having a low accuracy and specificity $[16,17]$. This is probably the reason why our cohort includes an important number of patients with preoperative minor GERD symptoms and associated hiatal hernia; furthermore, for the same reason, we had a considerable number of patients with postoperative de novo/aggravated GERD, who were treated with proton-pump inhibitors for at least 3 months after surgery, few of them having their sleeve converted to LGB due to severe reflux disease. 
The immediate postoperative general morbidity reported by us is within the accepted limits in the literature $[1,5,18]$; we note here the higher incidence of postoperative hemorrhage, most of which have required reintervention for hemostasis. We rarely have been able to clearly identify the source of hemorrhage, and the operation resumed to lavage and evacuation of the perigastric intraperitoneal hematoma. Although we did not statistically analyze this particular aspect, we did notice an increased frequency of bleeding in the group of patients with no oversewing, which actually corresponds to the opinion of several authors on the role of this reinforcement method in preventing hemorrhage at the level of the staple line $[5,19]$. We also recorded a proximal gastric leak that debuted at 6 days postoperatively; the reintervention revealed an $a b$ scess at the level of the upper gastric pole, which was laparoscopically drained and a feeding jejunostomy was performed. This leak did not close 4 months after surgery, and was finally solved through an endoscopic approach. The reported mortality is very low and concerns a single case, where an LSG was performed in our service; about 6 months after bariatric surgery, on the background of repeated admissions to pneumonia, a left pleural abscess was diagnosed and the patient was admitted to a thoracic surgery clinic. Evacuation of the pleural abscess is performed through left thoracotomy, yet it extends transdiaphragmatically, to the upper abdomen, confirming intraoperatively a left gastro-pleural fistula. Death occurs due to septic complications and multiple organ failure syndrome. Such rare but potentially fatal complications have been described after LSG, but also after other types of interventions; the importance of reintervention when the fistula is symptomatic is emphasized, regardless of the time elapsed since the primary bariatric procedure, with the need for an en-bloc resection of the fistula with total gastrectomy and reconstruction through an esophagojejunoanastomosis [20,21].

Although LSG is credited with excellent results, both in terms of weight reduction and improvement of the main comorbidities [22, 23], more studies with medium- and long-term follow-up describe a tendency for relapse with weight regain, after variable intervals of time from primary surgery $[1,5]$. We also noted this aspect in our studied group, where this trend becomes evident after approximately 2 years of follow-up. Concerning weight reduction, we also observed some correlations in the studied group; first we confirm results previously published by us [6] and also noted by others [24], where the elderly group (over 45 years) exhibits a significantly smaller weight loss. In this study, however, a negative correlation was also described for both the initial BMI and gender, with a significantly better weight loss in the low BMI (under 45 ) group and in the female group. In this respect our results are at odds with those of other authors, who describe superior results in terms of reducing weight in males but no differences in terms of influence on comorbidities [25].

Our study group included patients with many comorbidities, which is in fact not surprising in a country like Romania, where preventive medicine is just starting to develop; similarly to others $[1,4,5]$ we also described resolution or improvement of these comorbidities at 1 year after LSG and during follow-up. The lower percentage of preoperative OSAS found in our group can be attributed to the fact that not all patients underwent a polysomnography examination at the beginning of our study, so this pathology was certainly underdiagnosed. However, for those who had this preoperative investigation, repeated polysomnography within 1 year after LSG demonstrated a resolution/improvement of OSAS in $70.4 \%$ of cases.

This study has some limitations, particularly as regards the diversity in several technical characteristics: the number of trocars used, the performance of oversewing, the starting point of resection and thus the volume of the resected stomach. Therefore, our results might be biased due to these modifications of the surgical technique during the study period. Similarly, we were not able to perform in all patients several preoperative investigations, especially as concern GERD and OSAS. We included in the weightloss analysis five patients with single stage revision from gastric band to sleeve; there is evidence that revisional procedures result in different outcomes. Furthermore, the impact of surgery on several comorbidities, e.g. T2DM, was assessed mainly by changes in therapy and not by specific laboratory determinations (e.g. $\mathrm{HbA}_{1 c}$ ). All of these factors could possibly introduce a certain amount of bias.

\section{Conclusions}

Based on reported data we could consider LSG as a safe and effective technique; long-term follow-up 
reveals a tendency to weight regain after approximately 2 years from primary surgery, with the need for revisional surgery in some of the cases. The LSG is a more effective procedure in certain groups of patients (e.g. young women with an initially lower BMI). The present study contributes information on the situation of obesity management in Romania; indeed, with a significant overweight and obese population percentage, with an alarming increase in obesity-related diabetes and a corresponding budget effort, our country still fails to find resources to implement a national bariatric program, to address both prevention and treatment of this epidemic of our century.

\section{Conflict of interest}

The authors declare no conflict of interest.

\section{References}

1. Janik MR, Rogula TG, Mustafa RR, et al. Setting realistic expectation for weight loss after laparoscopic sleeve gastrectomy. Videosurgery Miniinv DOI: https://doi.org/10.5114/ wiitm.2019.81661

2. Himpens J, Dapri G, Cadière GB. A prospective randomized study between laparoscopic gastric banding and laparoscopic isolated sleeve gastrectomy: results after 1 and 3 years. Obes Surg 2006; 16: 1450-6.

3. Kehagias I, Karamanokos SN, Argentou M, Kalfarentzos F. Randomized clinical trial of laparoscopic Roux-en-Y gastric bypass versus laparoscopic sleeve gastrectomy for the management of patients with $B M I<50 \mathrm{~kg} / \mathrm{m}^{2}$. Obes Surg 2011; 21: 1650-6.

4. Boza C, Salinas J, Salgado N, et al. Laparoscopic sleeve gastrectomy as a stand-alone procedure for morbid obesity: report of 1000 cases and 3-year follow-up. Obes Surg 2012; 22: 866-71.

5. Rosenthal RJ, Diaz AA, Arvidsson D, et al. International sleeve gastrectomy expert panel consensus statement: best practice guidelines based on experience of 12,000 cases. Surg Obes Relat Dis 2012; 8: 8-19.

6. Neagoe RM, Mureșan M, Bancu Ș, et al. Results of laparoscopic sleeve gastrectomy-5-year follow-up study in an eastern European emerging bariatric center. Obesity Surgery 2016; 27: 983-9.

7. Givon-Maghala O, Spector R, Wasserberg N, et al. Technical aspects of laparoscopic sleeve gastrectomy in 25 morbidly obese patients. Obes Surg 2007; 17: 722-7.

8. Silecchia G, Boru C, Pecchia A, et al. Effectiveness of laparoscopic sleeve gastrectomy (first stage of biliopancreatic diversion with duodenal switch) on co-morbidities in super-obese high risk patients. Obes Surg 2006; 16: 1138-44.

9. Baltasar A, Serra C, Perez N, et al. Laparoscopic sleeve gastrec tomy: a multi-purpose bariatric operation. Obes Surg 2005; 15 1124-8.

10. Mognol P, Chosidow D, Marmuse JP. Laparoscopic sleeve gastrectomy as initial bariatric operation for high-risk patients: initial results in 10 patients. Obes Surg 2005; 15: 1030-3.
11. Choi YY, Bae J, Hur KY, et al. Reinforcing the staple line during laparoscopic sleeve gastrectomy: does it have advantages? A meta-analysis. Obes Surg 2012; 22: 1206-13.

12. Glaysher M, Khan OA, Mabvuure NT, et al. Laparoscopic line reinforcement during laparoscopic sleeve gastrectomy: does it affect clinical outcomes? Int J Surg 2013; 13: 286-9.

13. Kehagias I, Spyropoulos C, Karamanakos S, Kalfarentzos F. Efficacy of sleeve gastrectomy as sole procedure in patients with clinically severe obesity $\left(\mathrm{BMI} \leq 50 \mathrm{~kg} / \mathrm{m}^{2}\right)$. Surg Obes Relat Dis 2013; 9: 363-9.

14. Arman GA, Himpens J, Dhaenens J, et al. Long-term (11+ years) outcomes in weight, patient satisfaction, comorbidities, and gastroesophageal reflux treatment after laparoscopic sleeve gastrectomy. Surg Obes Relat Dis 2016; 12: 1778-86.

15. Mandeville Y, Van Looveren R, Vancoillie PJ, et al. Moderating the enthusiasm of sleeve gastrectomy: up to fifty percent of reflux symptoms after ten years in a consecutive series of one hundred laparoscopic sleeve gastrectomies. Obes Surg 2017; 27: 1797-803.

16. Althuwaini S, Bamehriz F, Aldohayan A, et al. Prevalence and predictors of gastroesophageal reflux disease after laparoscopic sleeve gastrectomy. Obes Surg 2018; 28: 916-22.

17. Genco A, Soricelli E, Casella G, et al. Gastroesophageal reflux disease and Barrett's esophagus after laparoscopic sleeve gastrectomy: a possible, underestimated longterm complication. Surg Obes Relat Dis 2017; 13: 568-74.

18. Bellanger DE, Greenway FL. Laparoscopic sleeve gastrectomy, 529 cases without a leak: short-term results and technical considerations. Obes Surg 2011; 21: 146-50.

19. Gentileschi P, Camperchioli I, D'Ugo S, et al. Staple-line reinforcement during laparoscopic sleeve gastrectomy using three different techniques: a randomized trial. Surg Endosc 2012; 26: 2623-9.

20. Nguyen D, Dip F, Hendricks L, et al. The surgical management of complex fistulas after sleeve gastrectomy. Obes Surg 2016; 26: 245-50.

21. Garcia-Quintero P, Hernandez-Murcia C, Romero R, et al. Gastropleural fistula after bariatric surgery: a report of two cases. J Robot Surg 2015; 9: 163-6.

22. Stefura T, Skomarovska O, Wysocki M, et al. Challenges associated with bariatric surgery - a multi-center report. Videosurgery Miniinv DOI: https://doi.org/10.5114/wiitm.2019.81370.

23. Wang MC, Guo XH, Zhang YW, et al. Laparoscopic RouX-en-Y gastric bypass versus sleeve gastrectomy for obese patients with type 2 diabetes: a meta-analysis of randomized controlled trials. Am Surg 2015; 81: 166-71.

24. Dowgiałto-Wnukiewicz N, Janik MR, Lech P, et al. Outcomes of sleeve gastrectomy in patients older than 60 years: a multicenter matched case-control study. Videosurgery Miniinv DOI: https://doi.org/10.5114/wiitm.2019.81450.

25. Perrone F, Bianciardi E, Benavoli D, et al. Gender influence on long-term weight loss and comorbidities after laparoscopic sleeve gastrectomy and Roux-en-Y gastric bypass: a prospective study with 5-year follow-up. Obes Surg 2016; 26: 276-81.

Received: 12.03.2019, accepted: 20.03.2019. 